\title{
A new sequence related to the Euler-Mascheroni constant
}

Shanhe $\mathrm{Wu}^{1 *}$ and Gabriel Bercu²

\section{"Correspondence:}

shanhewu@163.com

${ }^{1}$ Department of Mathematics, Longyan University, Longyan, P.R. China

Full list of author information is available at the end of the article

\begin{abstract}
In this paper, we provide a new quicker sequence convergent to the Euler-Mascheroni constant using an approximation of Padé type. Our sequence has a relatively simple form and higher speed of convergence. Moreover, we establish lower and upper bound estimates for the difference between the sequence and the Euler-Mascheroni constant.
\end{abstract}

MSC: 11Y60;41A60;41A25

Keywords: Sequences; Euler-Mascheroni constant; Rate of convergence; Lower and upper bounds

\section{Introduction}

The Euler-Mascheroni constant

$$
\gamma=0.5772156649015328 \ldots
$$

is one of the most famous constants in analysis and number theory. It is the limit of the sequence

$$
\gamma_{n}=1+\frac{1}{2}+\cdots+\frac{1}{n}-\log n
$$

There are many famous problems related to the properties of this constant; for example, it is not known yet whether the Euler-Mascheroni constant is a rational number. In recent years, many researchers made great efforts in the area of concerning the rate of convergence of the sequence $\left(\gamma_{n}\right)_{n \geq 1}$ and establishing sequences converging faster to the Euler-Mascheroni constant $\gamma$.

We begin with a brief overview of the relevant research.

To reveal the speed of convergence of the sequence $\left(\gamma_{n}\right)_{n \geq 1}$, Boas [5] and Mortici and Vernescu $[20,21]$ established the following double inequality for the difference between the sequence and the Euler-Mascheroni constant:

$$
\frac{1}{2 n+1}<\gamma_{n}-\gamma<\frac{1}{2 n}
$$

(c) The Author(s) 2018. This article is distributed under the terms of the Creative Commons Attribution 4.0 International License (http://creativecommons.org/licenses/by/4.0/), which permits unrestricted use, distribution, and reproduction in any medium, provided you give appropriate credit to the original author(s) and the source, provide a link to the Creative Commons license, and indicate if changes were made. 
DeTemple [12] modified the logarithmic term of $\gamma_{n}$ and showed that the sequence

$$
R_{n}=1+\frac{1}{2}+\cdots+\frac{1}{n}-\log \left(n+\frac{1}{2}\right)
$$

converges to $\gamma$ with rate of convergence $n^{-2}$, since

$$
\frac{1}{24(n+1)^{2}}<R_{n}-\gamma<\frac{1}{24 n^{2}} \text {. }
$$

Vernescu [28] provided the sequence

$$
V_{n}=1+\frac{1}{2}+\cdots+\frac{1}{n-1}+\frac{1}{2 n}-\log n,
$$

which also converges to $\gamma$ with rate of convergence $n^{-2}$, since

$$
\frac{1}{12(n+1)^{2}}<\gamma-V_{n}<\frac{1}{12 n^{2}} \text {. }
$$

Cristea and Mortici [11] introduced the family of sequences

$$
v_{n}(a, b)=1+\frac{1}{2}+\cdots+\frac{1}{n-2}+\frac{a n+b}{n(n-1)}-\log n,
$$

where $a, b$ are real parameters. Furthermore, they proved that, among the sequences $\left(v_{n}(a, b)\right)_{n \geq 1}$, the privileged one $\left(v_{n}\left(\frac{3}{2},-\frac{5}{12}\right)\right)_{n \geq 1}$ offers the best approximation to $\gamma$, since it has the rate of convergence $n^{-3}$. More precisely, for

$$
v_{n}\left(\frac{3}{2},-\frac{5}{12}\right)=1+\frac{1}{2}+\cdots+\frac{1}{n-2}+\frac{13}{12(n-1)}+\frac{5}{12 n}-\log n,
$$

they obtained the bounds

$$
\frac{1}{12 n^{3}}+\frac{11}{120 n^{4}}<v_{n}\left(\frac{3}{2},-\frac{5}{12}\right)-\gamma<\frac{1}{12 n^{3}}+\frac{13}{120 n^{4}} \quad(n \geq 9) .
$$

Lu [16] used continued fraction approximation to obtain the following faster sequence converging to the Euler-Mascheroni constant:

$$
r_{n}^{(2)}\left(\frac{1}{2}, \frac{1}{6}\right)=1+\frac{1}{2}+\cdots+\frac{1}{n}-\frac{3}{6 n+1}-\log n,
$$

which satisfies

$$
\frac{1}{72(n+1)^{3}}<\gamma-r_{n}^{(2)}\left(\frac{1}{2}, \frac{1}{6}\right)<\frac{1}{72 n^{3}} .
$$

Recently, Wu and Bercu [29] constructed the new sequence

$$
\omega_{n}=\sum_{k=1}^{n} \frac{1+\left(2 b_{1}-1\right)(-1)^{k-1}}{k}-\log \left[n+\frac{(-1)^{n-1}\left(2 b_{1}-1\right)+1}{2}\right]-\left(2 b_{1}-1\right) \log 2,
$$

which converges to $\gamma$ with rate of convergence $n^{-2}$. 
For more detail about the approximation of the Euler-Mascheroni constant with very high accuracy, we mention the works of Lu [16-18], Sweeney [27], Bailey [2], Crînganu [10], and Alzer and Koumandos [1]. We also mention the excellent survey by Lagarias [15]. $\mathrm{Hu}$ and Mortici $[13,14,19]$ provided some similar methods to deal with approximation of the constant $e$.

In this paper, starting from the sequence $\left(\gamma_{n}\right)_{n \geq 1}$, we use an approximation of Padé type and provide a new convergent sequence for Euler-Mascheroni constant.

The Padé approximant is the best approximation of a function by a rational function and often gives better approximation of the function than truncating its Taylor series. For these reasons, Padé approximants are also used in computer calculations (see $[3,30]$ ).

Recall the Padé approximant of $P(n)$ of order [1/2]:

$$
P_{[1 / 2]}(n)=\frac{\alpha_{0}+\alpha_{1} n}{1+\beta_{1} n+\beta_{2} n^{2}}=\frac{a_{1}}{n+b_{1}}+\frac{a_{2}}{n+b_{2}} .
$$

We will use this Padé approximant $p_{[1 / 2]}(n)$ as an additional term to establish a new quicker sequence converging to the Euler-Mascheroni constant. More precisely, we consider the following sequence:

$$
\Gamma_{n}^{(2)}=1+\frac{1}{2}+\cdots+\frac{1}{n}-\log n-\frac{a_{1}}{n+b_{1}}-\frac{a_{2}}{n+b_{2}} .
$$

Furthermore, we will provide lower and upper bound estimates for the difference between the sequence and the Euler-Mascheroni constant.

\section{Main results}

Our main results are stated in the following theorem.

Theorem 2.1 Let

$$
\Gamma_{n}^{(2)}=1+\frac{1}{2}+\cdots+\frac{1}{n}-\log n-\frac{a_{1}}{n+b_{1}}-\frac{a_{2}}{n+b_{2}},
$$

and let

$$
\begin{aligned}
& a_{1}=\frac{1}{24 b_{1}\left(1-3 b_{1}\right)}, \\
& a_{2}=-\frac{\left(6 b_{1}-1\right)^{2}}{24 b_{1}\left(1-3 b_{1}\right)}, \\
& b_{2}=\frac{b_{1}}{6 b_{1}-1}, \quad b_{1} \in\left(\frac{1}{6}, \frac{1}{3}\right) \cup\left(\frac{1}{3},+\infty\right) .
\end{aligned}
$$

Then we have the asymptotic expansion

$$
\Gamma_{n}^{(2)}=\gamma+\sum_{k=4}^{m}\left(-\frac{B_{k}}{k}+(-1)^{k}\left(a_{1} b_{1}^{k-1}+a_{2} b_{2}^{k-1}\right)\right) \frac{1}{n^{k}}+O\left(\frac{1}{n^{m+1}}\right)
$$

as $n \rightarrow \infty$, where $B_{k}$ are Bernoulli numbers. More explicitly, we have

$$
\Gamma_{n}^{(2)}=\gamma+\frac{1-10 p}{120} \cdot \frac{1}{n^{4}}+\frac{p^{2}}{2} \cdot \frac{1}{n^{5}}+\left(\frac{p^{2}-36 p^{3}}{12}-\frac{1}{252}\right) \frac{1}{n^{6}}+p^{3}(18 p-1) \frac{1}{n^{7}}
$$


Wu and Bercy Journal of Inequalities and Applications <wide>(2018) 2018:151

Page 4 of 12

$$
+\cdots+\left(\frac{\left(3-\sqrt{9-p^{-1}}\right)^{m-3}-\left(3+\sqrt{9-p^{-1}}\right)^{m-3}}{24(-1)^{m} p^{3-m} \sqrt{9-p^{-1}}}-\frac{B_{m}}{m}\right) \frac{1}{n^{m}}+O\left(\frac{1}{n^{m+1}}\right)
$$

as $n \rightarrow \infty$, where $p=b_{1}^{2} /\left(6 b_{1}-1\right)$.

Furthermore, we have the following double inequality:

$$
\frac{1-10 p}{120} \cdot \frac{1}{n^{4}}<\Gamma_{n}^{(2)}-\gamma<\frac{1-10 p}{120} \cdot \frac{1}{n^{4}}+\frac{p^{2}}{2} \cdot \frac{1}{n^{5}} .
$$

Proof Using the representation of the harmonic sum in terms of digamma function (see [4])

$$
1+\frac{1}{2}+\cdots+\frac{1}{n}=\gamma+\frac{1}{n}+\Psi(n)
$$

and the asymptotic formula

$$
\begin{aligned}
\Psi(z) & =\log z-\frac{1}{2 z}-\sum_{k=2}^{m} \frac{B_{2 k-2}}{(2 k-2) z^{2 k-2}}+O\left(\frac{1}{z^{2 m}}\right) \\
& =\log z-\frac{1}{2 z}-\frac{1}{12 z^{2}}+\frac{1}{120 z^{4}}-\frac{1}{252 z^{6}}+\cdots+\frac{-B_{2 m-2}}{(2 m-2) z^{2 m-2}}+O\left(\frac{1}{z^{2 m}}\right)
\end{aligned}
$$

we obtain

$$
\begin{aligned}
1 & +\frac{1}{2}+\cdots+\frac{1}{n}-\log n \\
& =\gamma+\frac{1}{n}+\Psi(n)-\log n \\
& =\gamma+\frac{1}{2 n}-\frac{1}{12 n^{2}}+\frac{1}{120 n^{4}}-\frac{1}{252 n^{6}}+\cdots+\frac{-B_{2 m-2}}{(2 m-2) n^{2 m-2}}+O\left(\frac{1}{n^{2 m}}\right) .
\end{aligned}
$$

Hence

$$
\begin{aligned}
\Gamma_{n}^{(2)}= & 1+\frac{1}{2}+\cdots+\frac{1}{n}-\log n-\frac{a_{1}}{n+b_{1}}-\frac{a_{2}}{n+b_{2}} \\
= & \gamma-\frac{a_{1}}{n+b_{1}}-\frac{a_{2}}{n+b_{2}}+\frac{1}{2 n}-\frac{1}{12 n^{2}}+\frac{1}{120 n^{4}} \\
& -\frac{1}{252 n^{6}}+\cdots+\frac{-B_{2 m-2}}{(2 m-2) n^{2 m-2}}+O\left(\frac{1}{n^{2 m}}\right) .
\end{aligned}
$$

Using the power series expansion gives

$$
\begin{aligned}
\frac{a_{1}}{n+b_{1}} & =\frac{a_{1}}{n}\left(\frac{1}{1+\frac{b_{1}}{n}}\right) \\
& =\frac{a_{1}}{n}\left(1-\frac{b_{1}}{n}+\frac{b_{1}^{2}}{n^{2}}-\frac{b_{1}^{3}}{n^{3}}+\cdots+(-1)^{m} \frac{b_{1}^{m}}{n^{m}}\right)+O\left(\frac{1}{n^{m+2}}\right) \\
& =\frac{a_{1}}{n}-\frac{a_{1} b_{1}}{n^{2}}+\frac{a_{1} b_{1}^{2}}{n^{3}}-\frac{a_{1} b_{1}^{3}}{n^{4}}+\cdots+(-1)^{m} \frac{a_{1} b_{1}^{m}}{n^{m+1}}+O\left(\frac{1}{n^{m+2}}\right)
\end{aligned}
$$


and

$$
\frac{a_{2}}{n+b_{2}}=\frac{a_{2}}{n}-\frac{a_{2} b_{2}}{n^{2}}+\frac{a_{2} b_{2}^{2}}{n^{3}}-\frac{a_{2} b_{2}^{3}}{n^{4}}+\cdots+(-1)^{m} \frac{a_{2} b_{2}^{m}}{n^{m+1}}+O\left(\frac{1}{n^{m+2}}\right)
$$

as $n \rightarrow \infty$. Thus we obtain

$$
\begin{aligned}
\Gamma_{n}^{(2)}= & \gamma+\left(\frac{1}{2}-a_{1}-a_{2}\right) \frac{1}{n}+\left(-\frac{1}{12}+a_{1} b_{1}+a_{2} b_{2}\right) \frac{1}{n^{2}}-\left(a_{1} b_{1}^{2}+a_{2} b_{2}^{2}\right) \frac{1}{n^{3}} \\
& +\left(\frac{1}{120}+a_{1} b_{1}^{3}+a_{2} b_{2}^{3}\right) \frac{1}{n^{4}}-\left(a_{1} b_{1}^{4}+a_{2} b_{2}^{4}\right) \frac{1}{n^{5}}+\left(-\frac{1}{252}+a_{1} b_{1}^{5}+a_{2} b_{2}^{5}\right) \frac{1}{n^{6}} \\
& +\cdots+\left(\frac{-B_{2 m-2}}{2 m-2}+a_{1} b_{1}^{2 m-3}+a_{2} b_{2}^{2 m-3}\right) \frac{1}{n^{2 m-2}} \\
& +\left(-a_{1} b_{1}^{2 m-2}-a_{2} b_{2}^{2 m-2}\right) \frac{1}{n^{2 m-1}}+O\left(\frac{1}{n^{2 m}}\right) .
\end{aligned}
$$

From the assumption conditions

$$
\begin{aligned}
& a_{1}=\frac{1}{24 b_{1}\left(1-3 b_{1}\right)}, \\
& a_{2}=-\frac{\left(6 b_{1}-1\right)^{2}}{24 b_{1}\left(1-3 b_{1}\right)}, \\
& b_{2}=\frac{b_{1}}{6 b_{1}-1}, \quad b_{1} \in\left(\frac{1}{6}, \frac{1}{3}\right) \cup\left(\frac{1}{3},+\infty\right),
\end{aligned}
$$

we have

$$
\frac{1}{2}-a_{1}-a_{2}=0, \quad-\frac{1}{12}+a_{1} b_{1}+a_{2} b_{2}=0, \quad a_{1} b_{1}^{2}+a_{2} b_{2}^{2}=0 .
$$

Therefore

$$
\begin{aligned}
\Gamma_{n}^{(2)}= & \gamma+\left(\frac{1}{120}+a_{1} b_{1}^{3}+a_{2} b_{2}^{3}\right) \frac{1}{n^{4}}-\left(a_{1} b_{1}^{4}+a_{2} b_{2}^{4}\right) \frac{1}{n^{5}}+\left(-\frac{1}{252}+a_{1} b_{1}^{5}+a_{2} b_{2}^{5}\right) \frac{1}{n^{6}} \\
& +\cdots+\left(\frac{-B_{2 m-2}}{2 m-2}+a_{1} b_{1}^{2 m-3}+a_{2} b_{2}^{2 m-3}\right) \frac{1}{n^{2 m-2}} \\
& +\left(-a_{1} b_{1}^{2 m-2}-a_{2} b_{2}^{2 m-2}\right) \frac{1}{n^{2 m-1}}+O\left(\frac{1}{n^{2 m}}\right) .
\end{aligned}
$$

Note that, for all odd Bernoulli numbers $B_{2 m-1}=0(m \geq 2)$, the last expression can be rewritten as

$$
\begin{aligned}
\Gamma_{n}^{(2)}= & \gamma+\left(\frac{1}{120}+a_{1} b_{1}^{3}+a_{2} b_{2}^{3}\right) \frac{1}{n^{4}}+\cdots+\left(\frac{-B_{2 m-2}}{2 m-2}+a_{1} b_{1}^{2 m-3}+a_{2} b_{2}^{2 m-3}\right) \frac{1}{n^{2 m-2}} \\
& +\left(\frac{-B_{2 m-1}}{2 m-1}-a_{1} b_{1}^{2 m-2}-a_{2} b_{2}^{2 m-2}\right) \frac{1}{n^{2 m-1}}+O\left(\frac{1}{n^{2 m}}\right)
\end{aligned}
$$

and

$$
\Gamma_{n}^{(2)}=\gamma+\left(\frac{1}{120}+a_{1} b_{1}^{3}+a_{2} b_{2}^{3}\right) \frac{1}{n^{4}}+\cdots+\left(\frac{-B_{2 m-3}}{2 m-3}+a_{1} b_{1}^{2 m-4}+a_{2} b_{2}^{2 m-4}\right) \frac{1}{n^{2 m-3}}
$$




$$
+\left(\frac{-B_{2 m-2}}{2 m-2}+a_{1} b_{1}^{2 m-3}+a_{2} b_{2}^{2 m-3}\right) \frac{1}{n^{2 m-2}}+O\left(\frac{1}{n^{2 m-1}}\right)
$$

that is,

$$
\Gamma_{n}^{(2)}=\gamma+\sum_{k=4}^{m}\left(-\frac{B_{k}}{k}+(-1)^{k}\left(a_{1} b_{1}^{k-1}+a_{2} b_{2}^{k-1}\right)\right) \frac{1}{n^{k}}+O\left(\frac{1}{n^{m+1}}\right)
$$

which is the desired Eq. (2.1) in Theorem 2.1.

On the other hand, from

$$
p=\frac{b_{1}^{2}}{6 b_{1}-1}, \quad b_{2}=\frac{b_{1}}{6 b_{1}-1}, \quad b_{1} \in\left(\frac{1}{6}, \frac{1}{3}\right) \cup\left(\frac{1}{3},+\infty\right),
$$

we have $b_{1} b_{2}=p, b_{1}+b_{2}=6 p\left(p>\frac{1}{9}\right)$, which implies that $b_{1}$ and $b_{2}$ are the roots of the equation $x^{2}-6 p x+p=0$. Therefore,

$$
b_{1,2}=3 p \pm \sqrt{9 p^{2}-p} \text {. }
$$

It is easy to observe that

$$
\begin{aligned}
& a_{1}=\frac{1}{24 b_{1}\left(1-3 b_{1}\right)}=\frac{b_{2}}{12 b_{1}\left(b_{2}-b_{1}\right)}, \\
& a_{2}=-\frac{\left(6 b_{1}-1\right)^{2}}{24 b_{1}\left(1-3 b_{1}\right)}=\frac{-b_{1}}{12 b_{2}\left(b_{2}-b_{1}\right)},
\end{aligned}
$$

and thus

$$
\begin{aligned}
a_{1} b_{1}^{k-1}+a_{2} b_{2}^{k-1} & =\frac{b_{2} b_{1}^{k-1}}{12 b_{1}\left(b_{2}-b_{1}\right)}-\frac{b_{1} b_{2}^{k-1}}{12 b_{2}\left(b_{2}-b_{1}\right)} \\
& =-\frac{b_{1} b_{2}\left(b_{1}^{k-3}-b_{2}^{k-3}\right)}{12\left(b_{1}-b_{2}\right)} \\
& =\frac{\left(3-\sqrt{9-p^{-1}}\right)^{k-3}-\left(3+\sqrt{9-p^{-1}}\right)^{k-3}}{24 p^{3-k} \sqrt{9-p^{-1}}} .
\end{aligned}
$$

It follows from (2.6) that

$$
\begin{aligned}
\Gamma_{n}^{(2)} & =\gamma+\sum_{k=4}^{m}\left(-\frac{B_{k}}{k}+(-1)^{k}\left(a_{1} b_{1}^{k-1}+a_{2} b_{2}^{k-1}\right)\right) \frac{1}{n^{k}}+O\left(\frac{1}{n^{m+1}}\right) \\
& =\gamma+\sum_{k=4}^{m}\left(-\frac{B_{k}}{k}+\frac{\left(3-\sqrt{9-p^{-1}}\right)^{k-3}-\left(3+\sqrt{9-p^{-1}}\right)^{k-3}}{24(-1)^{k} p^{3-k} \sqrt{9-p^{-1}}}\right) \frac{1}{n^{k}}+O\left(\frac{1}{n^{m+1}}\right),
\end{aligned}
$$

which implies the desired Eq. (2.2) in Theorem 2.1.

Next, we will show the double inequality (2.3). We define the sequences $\left(z_{n}\right)_{n \geq 1}$ and $\left(u_{n}\right)_{n \geq 1}$ by

$$
z_{n}=\Gamma_{n}^{(2)}-\gamma-\frac{1-10 p}{120} \cdot \frac{1}{n^{4}}
$$


and

$$
u_{n}=\Gamma_{n}^{(2)}-\gamma-\frac{1-10 p}{120} \cdot \frac{1}{n^{4}}-\frac{p^{2}}{2} \cdot \frac{1}{n^{5}} .
$$

It follows from (2.2) that

$$
\begin{aligned}
& \Gamma_{n}^{(2)}-\gamma-\frac{1-10 p}{120} \cdot \frac{1}{n^{4}}=O\left(\frac{1}{n^{5}}\right), \\
& \Gamma_{n}^{(2)}-\gamma-\frac{1-10 p}{120} \cdot \frac{1}{n^{4}}-\frac{p^{2}}{2} \cdot \frac{1}{n^{5}}=O\left(\frac{1}{n^{6}}\right),
\end{aligned}
$$

and thus we have

$$
\lim _{n \rightarrow \infty} z_{n}=0 \quad \text { and } \quad \lim _{n \rightarrow \infty} u_{n}=0 .
$$

To prove that $z_{n}>0$ and $u_{n}<0$ for $n \geq 1$, it suffices to show that $\left(z_{n}\right)_{n \geq 1}$ is decreasing and $\left(u_{n}\right)_{n \geq 1}$ is increasing.

Let

$$
\begin{aligned}
& z_{n+1}-z_{n}=f(n), \\
& u_{n+1}-u_{n}=g(n),
\end{aligned}
$$

where

$$
\begin{aligned}
f(x)= & \frac{1}{x+1}+\log x-\log (x+1)+a_{1}\left(\frac{1}{x+b_{1}}-\frac{1}{x+b_{1}+1}\right) \\
& +a_{2}\left(\frac{1}{x+b_{2}}-\frac{1}{x+b_{2}+1}\right)+\frac{10 p-1}{120}\left(\frac{1}{(x+1)^{4}}-\frac{1}{x^{4}}\right), \\
g(x)= & f(x)-\frac{p^{2}}{2}\left(\frac{1}{(x+1)^{5}}-\frac{1}{x^{5}}\right), \quad x \in[1,+\infty) .
\end{aligned}
$$

It is easy to verify that

$$
\frac{a_{1}}{x+b_{1}}+\frac{a_{2}}{x+b_{2}}=\frac{6 x+36 p-1}{12\left(x^{2}+6 p x+p\right)}
$$

and

$$
\frac{a_{1}}{x+b_{1}+1}+\frac{a_{2}}{x+b_{2}+1}=\frac{6 x+36 p+5}{12\left(x^{2}+2 x++6 p x+1+7 p\right)} .
$$

Hence

$$
\begin{aligned}
f(x)= & \frac{1}{x+1}+\log x-\log (x+1)+\frac{6 x+36 p-1}{12\left(x^{2}+6 p x+p\right)} \\
& -\frac{6 x+36 p+5}{12\left(x^{2}+2 x++6 p x+1+7 p\right)}+\frac{10 p-1}{120}\left(\frac{1}{(x+1)^{4}}-\frac{1}{x^{4}}\right) .
\end{aligned}
$$


Differentiating $f(x)$ with respect to $x$ gives

$$
f^{\prime}(x)=\frac{P(x)}{30 x^{5}(x+1)^{5}\left(x^{2}+6 p x+p\right)^{2}\left(x^{2}+2 x+6 p x+1+7 p\right)^{2}},
$$

where

$$
\begin{aligned}
& P(x)=450 p^{2} x^{11}+\left(7020 p^{2}\left(p-\frac{1}{9}\right)+3360 p\left(p-\frac{1}{9}\right)+\frac{3360}{9}\left(p-\frac{1}{9}\right)+\frac{2955}{81}\right) x^{10} \\
& +\left(36,720 p^{3}\left(p-\frac{1}{9}\right)+41,820 p^{2}\left(p-\frac{1}{9}\right)+\frac{102,570}{9} p\left(p-\frac{1}{9}\right)\right. \\
& \left.+\frac{92,850}{81}\left(p-\frac{1}{9}\right)+\frac{74,625}{729}\right) x^{9}+\left(64,800 p^{4}\left(p-\frac{1}{9}\right)+191,520 p^{3}\left(p-\frac{1}{9}\right)\right. \\
& +\frac{1,018,260}{9} p^{2}\left(p-\frac{1}{9}\right)+\frac{1,798,290}{81} p\left(p-\frac{1}{9}\right)+\frac{1,390,050}{729}\left(p-\frac{1}{9}\right) \\
& \left.+\frac{1,055,439}{6561}\right) x^{8} \\
& +\left(302,400 p^{4}\left(p-\frac{1}{9}\right)+446,910 p^{3}\left(p-\frac{1}{9}\right)+\frac{1,619,250}{9} p^{2}\left(p-\frac{1}{9}\right)\right. \\
& \left.+\frac{2,158,710}{81} p\left(p-\frac{1}{9}\right)+\frac{1,383,054}{729}\left(p-\frac{1}{9}\right)+\frac{1,028,760}{6561}\right) x^{7} \\
& +\left(615,600 p^{4}\left(p-\frac{1}{9}\right)+612,290 p^{3}\left(p-\frac{1}{9}\right)+\frac{1,632,350}{9} p^{2}\left(p-\frac{1}{9}\right)\right. \\
& \left.+\frac{1,634,294}{81} p\left(p-\frac{1}{9}\right)+\frac{863,012}{729}\left(p-\frac{1}{9}\right)+\frac{659,621}{6561}\right) x^{6} \\
& +\left(724,800 p^{4}\left(p-\frac{1}{9}\right)+\frac{4,803,690}{9} p^{3}\left(p-\frac{1}{9}\right)+\frac{9,579,126}{81} p^{2}\left(p-\frac{1}{9}\right)\right. \\
& \left.+\frac{7,355,676}{729} p\left(p-\frac{1}{9}\right)+\frac{3,484,686}{6561}\left(p-\frac{1}{9}\right)+\frac{2,953,245}{59,049}\right) x^{5} \\
& +\left(536,210 p^{4}\left(p-\frac{1}{9}\right)+\frac{2,700,431}{9} p^{3}\left(p-\frac{1}{9}\right)+\frac{4,053,779}{81} p^{2}\left(p-\frac{1}{9}\right)\right. \\
& \left.+\frac{2,169,314}{729} p\left(p-\frac{1}{9}\right)+\frac{962,090}{6561}\left(p-\frac{1}{9}\right)+\frac{903,041}{59,049}\right) x^{4} \\
& +\left(247,060 p^{4}\left(p-\frac{1}{9}\right)+\frac{968,266}{9} p^{3}\left(p-\frac{1}{9}\right)+\frac{1,113,742}{81} p^{2}\left(p-\frac{1}{9}\right)\right. \\
& \left.+\frac{437,230}{729} p\left(p-\frac{1}{9}\right)+\frac{240,400}{6561}\left(p-\frac{1}{9}\right)+\frac{240,400}{59,049}\right) x^{3}+\left(66,580 p^{4}\left(p-\frac{1}{9}\right)\right. \\
& +\frac{213,658}{9} p^{3}\left(p-\frac{1}{9}\right)+\frac{195,838}{81} p^{2}\left(p-\frac{1}{9}\right)+\frac{58,786}{729} p\left(p-\frac{1}{9}\right) \\
& \left.+\frac{45,664}{6561}\left(p-\frac{1}{9}\right)+\frac{45,664}{59,049}\right) x^{2} \\
& +\left(9170 p^{4}\left(p-\frac{1}{9}\right)+\frac{25,937}{9} p^{3}\left(p-\frac{1}{9}\right)+\frac{20,429}{81} p^{2}\left(p-\frac{1}{9}\right)+\frac{5120}{729} p^{2}\right) x \\
& +\left(490 p^{4}\left(p-\frac{1}{9}\right)+\frac{1309}{9} p^{3}\left(p-\frac{1}{9}\right)+\frac{985}{81} p^{2}\left(p-\frac{1}{9}\right)+\frac{256}{729} p^{2}\right) \text {. }
\end{aligned}
$$


Since $p>\frac{1}{9}$, we have $P(x)>0$, which implies that $f^{\prime}(x)>0$ for $x \in[1,+\infty)$. Hence $f(x)$ is strictly increasing on $[1,+\infty)$. It follows from $\lim _{x \rightarrow+\infty} f(x)=0$ that $f(x)<0$ for $x \in[1,+\infty)$. This yields $z_{n+1}-z_{n}=f(n)<0$, so that $\left(z_{n}\right)_{n \geq 1}$ is strictly decreasing, which, along with $\lim _{n \rightarrow \infty} z_{n}=0$, leads us to $z_{n}>0$. The left-hand inequality of (2.3) is proved.

Similarly, differentiating $g(x)$ with respect to $x$, we obtain

$$
g^{\prime}(x)=\frac{-Q(x)}{30 x^{6}(x+1)^{6}\left(x^{2}+6 p x+p\right)^{2}\left(x^{2}+2 x+6 p x+1+7 p\right)^{2}},
$$

where

$$
\begin{aligned}
Q(x)= & \left(3780 p^{2}\left(p-\frac{1}{9}\right)+\frac{2835}{9} p^{2}+5\right) x^{12}+60,480 p^{3}\left(p-\frac{1}{9}\right) \\
& \left.+28,560 p^{2}\left(p-\frac{1}{9}\right)+\frac{22,890}{9} p^{2}+120 p+30\right) x^{11}+\left(324,000 p^{4}\left(p-\frac{1}{9}\right)\right. \\
& +381,960 p^{3}\left(p-\frac{1}{9}\right)+\frac{916,560}{9} p^{2}\left(p-\frac{1}{9}\right)+\frac{859,455}{81} p^{2}+680 p \\
& +76) x^{10}+\left(583,200 p^{6}+1,771,200 p^{5}+927,870 p^{4}+105,180 p^{3}\right. \\
& \left.+2610 p^{2}+1624 p+105\right) x^{9}+\left(3,013,200 p^{6}+4,492,800 p^{5}\right. \\
& \left.+1,555,150 p^{4}+138,450 p^{3}+9066 p^{2}+2122 p+85\right) x^{8}+\left(7,095,600 p^{6}\right. \\
& +7,020,600 p^{5}+1,794,700 p^{4}+138,504 p^{3}+12,426 p^{2}+1648 p \\
& +40) x^{7}+\left(10,103,400 p^{6}+7,393,390 p^{5}+1,467,221 p^{4}+100,436 p^{3}\right. \\
& \left.+9010 p^{2}+774 p+10\right) x^{6}+\left(9,461,250 p^{6}+5,365,230 p^{5}+846,847 p^{4}\right. \\
& \left.+49,296 p^{3}+4263 p^{2}+214 p+1\right) x^{5}+\left(5,874,525 p^{6}+2,667,310 p^{5}\right. \\
& \left.+337,249 p^{4}+15,224 p^{3}+1191 p^{2}+32 p\right) x^{4}+\left(2,352,300 p^{6}+883,050 p^{5}\right. \\
& \left.+89,495 p^{4}+2688 p^{3}+209 p^{2}+2 p\right) x^{3}+\left(568,125 p^{6}+183,690 p^{5}\right. \\
& \left.+15,221 p^{4}+222 p^{3}+22 p^{2}\right) x^{2}+\left(72,450 p^{6}+21,410 p^{5}+1559 p^{4}\right. \\
& \left.+4 p^{3}+p^{2}\right) x+\left(3675 p^{6}+1050 p^{5}+75 p^{4}\right) .
\end{aligned}
$$

Since $p>\frac{1}{9}$, we conclude that $Q(x)<0$. Thus we have $g^{\prime}(x)<0$ for $x \in[1,+\infty)$. It follows that $g(x)$ is strictly decreasing on $[1,+\infty)$. Since $\lim _{x \rightarrow+\infty} g(x)=0$, we have $g(x)>0$ for $x \in[1,+\infty)$. This yields $u_{n+1}-u_{n}=g(n)>0$, which implies that $\left(u_{n}\right)_{n \geq 1}$ is strictly increasing. We obtain $u_{n}<0$ since $\lim _{n \rightarrow \infty} u_{n}=0$. The right-hand inequality of (2.3) is proved.

This completes the proof of Theorem 2.1.

\section{Some remarks on Theorem 2.1}

Remark 3.1 Lu [16] constructed the sequence

$$
r_{n}^{(3)}=1+\frac{1}{2}+\cdots+\frac{1}{n}-\log n-\frac{a_{1}}{n+\frac{a_{2} \cdot n}{n+a_{3}}},
$$


where $a_{1}=\frac{1}{2}, a_{2}=\frac{1}{6}$, and $a_{3}=-\frac{1}{6}$ and proved the inequality

$$
\frac{1}{120(n+1)^{4}}<r_{n}^{(3)}-\gamma<\frac{1}{120(n-1)^{4}}
$$

In Theorem 2.1 , if we take $p=\frac{1}{5}$ in inequality (2.3), then we get

$$
\frac{1}{120 n^{4}}-\frac{1}{50 n^{5}}<\gamma-\Gamma_{n}^{(2)}<\frac{1}{120 n^{4}}
$$

Since

$$
\frac{1}{120(n+1)^{4}}<\frac{1}{120 n^{4}}-\frac{1}{50 n^{5}}
$$

and

$$
\frac{1}{120 n^{4}}<\frac{1}{120(n-1)^{4}}
$$

for all natural numbers $n \geq 5$, the sequence $\left(\Gamma_{n}^{(2)}\right)_{n \geq 1}$ provides a more accurate double inequality for the difference between the sequence and the Euler-Mascheroni constant than the sequence $\left(r_{n}^{(3)}\right)_{n \geq 1}$ from [16].

Remark 3.2 Lu et al. [18] considered the following sequence converging to the EulerMascheroni constant:

$$
r_{n, 2}^{(3)}=1+\frac{1}{2}+\cdots+\frac{1}{n}-\log n-\frac{1}{2} \log \left(1+\frac{a_{1}}{n+\frac{a_{2} \cdot n}{n+a_{3}}}\right)
$$

where $a_{1}=1, a_{2}=-\frac{1}{3}$, and $a_{3}=\frac{1}{3}$, and they proved that

$$
\frac{1}{180(n+1)^{4}}<\gamma-r_{n, 2}^{(3)}<\frac{1}{180 n^{4}}
$$

In Theorem 2.1, if we choose $p=\frac{1}{6}$ in inequality (2.3), then we obtain

$$
\frac{1}{180 n^{4}}-\frac{1}{72 n^{5}}<\gamma-\Gamma_{n}^{(2)}<\frac{1}{180 n^{4}}
$$

It is easy to find that

$$
\frac{1}{180(n+1)^{4}}<\frac{1}{180 n^{4}}-\frac{1}{72 n^{5}}
$$

for all natural numbers $n \geq 5$, so the sequence $\left(\Gamma_{n}^{(2)}\right)_{n \geq 1}$ improves inequality (3.5) from [18].

Remark 3.3 For more results relating to the Euler constant, sequences, and some estimates, we refer the interested reader to Sîntǎmărian [23-26] and the references therein. 


\section{Conclusion}

To provide a sequence converging faster to the Euler-Mascheroni constant, we construct a sequence $\Gamma_{n}^{(2)}$ by reference to the Padé approximant method, which improves the rate of convergence of the sequences introduced by $\mathrm{Lu}[16,18]$. Our sequence depends on a real parameter and has a relatively simple form. It is worth noting that the method mentioned is also applicable to establishing estimates of bounds for some special means. For example, the method can be used for further study on the results obtained previously by Chu et al. [6-9], Qian and Chu [22], Yang et al. [31-34], and Zhao et al. [35].

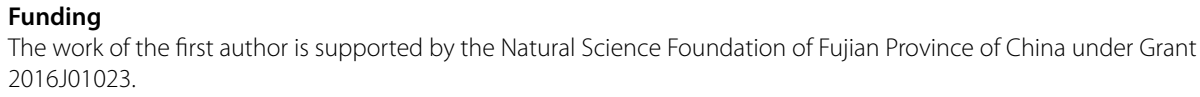

\section{Competing interests}

The authors declare that they have no competing interests.

\section{Authors' contributions}

Both authors read and approved the final manuscript.

\section{Author details}

'Department of Mathematics, Longyan University, Longyan, P.R. China. ${ }^{2}$ Department of Mathematics and Computer Sciences, "Dunărea de Jos" University of Galaţi, Galaţi, Romania.

\section{Publisher's Note}

Springer Nature remains neutral with regard to jurisdictional claims in published maps and institutional affiliations.

Received: 9 January 2018 Accepted: 4 June 2018 Published online: 28 June 2018

\section{References}

1. Alzer, H., Koumandos, S.: Series representations for $\gamma$ and other mathematical constants. Anal. Math. 34(1), 1-8 (2008)

2. Bailey, D.H.: Numerical results on the transcendence of constants involving $\pi$, $e$ and Euler's constant. Math. Comput. 50, 275-281 (1988)

3. Bercu, G., Wu, S.: Refinements of certain hyperbolic inequalities via the Padé approximation method. J. Nonlinear Sci. Appl. 9(7), 5011-5020 (2016)

4. Bernardo, J.M.: Algorithm AS 103: Psi (digamma) function. Appl. Stat. 25(3), 315-317 (1976)

5. Boas, R.P.: Estimating remainders. Math. Mag. 51(2), 83-89 (1978)

6. Chu, Y.M., Wang, M.K.: Optimal Lehmer mean bounds for the Toader mean. Results Math. 61(3-4), 223-229 (2012)

7. Chu, Y.M., Wang, M.K., Gong, W.M.: Two sharp double inequalities for Seiffert mean. J. Inequal. Appl. 2011, Article ID 44 (2011)

8. Chu, Y.M., Wang, M.K., Qiu, S.L.: Optimal combinations bounds of root-square and arithmetic means for Toader mean. Proc. Indian Acad. Sci. Math. Sci. 122(1), 41-51 (2012)

9. Chu, Y.M., Xia, W.F.: Two sharp inequalities for power mean, geometric mean, and harmonic mean. J. Inequal. Appl. 2009, Article 741923, 6 pages (2009)

10. Cringanu, J: Better bounds in Chen's inequalities for the Euler's constant. Bull. Aust. Math. Soc. 92(1), 94-97 (2015)

11. Cristea, V.G., Mortici, C.: Latter research on Euler-Mascheroni constant. arXiv:1312.4397 [math.CA] (2013)

12. DeTemple, D.W.: A quicker covergences to Euler's constant. Am. Math. Mon. 100(5), 468-470 (1993)

13. Hu, Y., Mortici, C.: Sharp inequalities related to the constant e. J. Inequal. Appl. 2014, Article ID 382 (2014)

14. Hu, Y., Mortici, C.: On the Keller limit and generalization. J. Inequal. Appl. 2016, Article ID 97 (2016)

15. Lagarias, J.C.: Euler's constant: Euler's work and modern developments. Bull. Am. Math. Soc. 50(4), 527-628 (2013)

16. Lu, D.: A new quicker sequence convergent to Euler's constant. J. Number Theory 136, 320-329 (2014)

17. Lu, D.: Some new improved classes of convergence towards Euler's constant. Appl. Math. Comput. 243, 24-32 (2014)

18. Lu, D., Song, L., Yu, Y.: Some new continued fraction approximation of Euler's constant. J. Number Theory 147, 69-80 (2015)

19. Mortici, C., Hu, Y:: On some convergences to the constant $e$ and improvements of Carleman's inequality. Carpath. J. Math. 31(2), 249-254 (2015)

20. Mortici, C., Vernescu, A.: An improvement of the convergence speed of the sequence $\left(\gamma_{n}\right)_{n \geq 1}$ converging to Euler's constant. An. Ştiinţ. Univ. 'Ovidius' Constanţa 13, 97-100 (2005)

21. Mortici, C., Vernescu, A.: Some new facts in discrete asymptotic analysis. Math. Balk. 21, 301-308 (2007)

22. Qian, W.M., Chu, Y.M.: Sharp bounds for a special quasi-arithmetic mean in terms of arithmetic and geometric means with two parameters. J. Inequal. Appl. 2017, Article ID 274 (2017)

23. Sîntămărian, A.: A generalization of Euler's constant. Numer. Algorithms 46(2), 141-151 (2007)

24. Sîntămărian, A.: Some new sequences that converge to a generalization of Euler's constant. Creative Math. Inform. 20(2), 191-196 (2011)

25. Sîntămărian, A.: New sequences that converge to a generalization of Euler's constant. Integers 11(2), 127-138 (2011)

26. Sîntǎmǎrian, A.: Euler's constant, sequences and some estimates. Surv. Math. Appl. 8, 103-114 (2013) 
27. Sweeney, D.W.: On the computation of Euler's constant. Math. Comput. 17, 170-178 (1963)

28. Vernescu, A.: A new accelerate convergence to the constant of Euler. Gaz. Mat., Ser. A 104(4), $273-278$ (1999) (in Romanian)

29. Wu, S., Bercu, G.: Fast convergence of generalized DeTemple sequences and the relation to the Riemann zeta function. J. Inequal. Appl. 2017, Article ID 110 (2017)

30. Wu, S., Bercu, G.: Padé approximants for inverse trigonometric functions and their applications. J. Inequal. Appl. 2017, Article ID 31 (2017)

31. Yang, Z.H., Chu, Y.M.: A monotonicity property involving the generalized elliptic integral of the first kind. Math. Inequal. Appl. 20(3), 729-735 (2017)

32. Yang, Z.H., Qian, W.M., Chu, Y.M., Zhang, W.: On rational bounds for the gamma function. J. Inequal. Appl. 2017, Article ID $210(2017)$

33. Yang, Z.H., Qian, W.M., Chu, Y.M., Zhang, W.: Monotonicity rule for the quotient of two functions and its application. J. Inequal. Appl. 2017, Article ID 106 (2017)

34. Yang, Z.H., Zhang, W., Chu, Y.M.: Sharp Gautschi inequality for parameter $0<p<1$ with applications. Math. Inequal. Appl. 20(4), 1107-1120 (2017)

35. Zhao, T.H., Chu, Y.M., Jiang, Y.P.: Monotonic and logarithmically convex properties of a function involving gamma functions. J. Inequal. Appl. 2009, Article ID 728612 (2009)

\section{Submit your manuscript to a SpringerOpen ${ }^{\circ}$} journal and benefit from:

- Convenient online submission

Rigorous peer review

- Open access: articles freely available online

- High visibility within the field

- Retaining the copyright to your article

Submit your next manuscript at $\gg$ springeropen.com 